\title{
A Use Case Methodology to Handle Conflicting Controller Requirements for Future Power Systems
}

\author{
Heussen, Kai; Uslar, Mathias; Tornelli, Carlo
}

Published in:

2015 International Symposium on Smart Electric Distribution Systems and Technologies (EDST)

Link to article, DOI:

10.1109/SEDST.2015.7315275

Publication date:

2015

Document Version

Peer reviewed version

Link back to DTU Orbit

Citation (APA):

Heussen, K., Uslar, M., \& Tornelli, C. (2015). A Use Case Methodology to Handle Conflicting Controller Requirements for Future Power Systems. In 2015 International Symposium on Smart Electric Distribution Systems and Technologies (EDST) (pp. 582-587). IEEE. https://doi.org/10.1109/SEDST.2015.7315275

\section{General rights}

Copyright and moral rights for the publications made accessible in the public portal are retained by the authors and/or other copyright owners and it is a condition of accessing publications that users recognise and abide by the legal requirements associated with these rights.

- Users may download and print one copy of any publication from the public portal for the purpose of private study or research.

- You may not further distribute the material or use it for any profit-making activity or commercial gain

- You may freely distribute the URL identifying the publication in the public portal 


\section{A Use Case Methodology to Handle Conflicting Controller Requirements for Future Power Systems}

\author{
Kai Heussen \\ Department of Electrical Engineering \\ Technical University of Denmark \\ Email: kh@elektro.dtu.dk
}

\author{
Mathias Uslar \\ OFFIS - Institute for Information Systems \\ Oldenburg, Germany \\ Email: uslar@offis.de
}

\author{
Carlo Tornelli \\ RSE SpA \\ Milano, Italy \\ Email: carlo.tornelli@rse-web.it
}

\begin{abstract}
This paper proposes a standards based requirements elicitation and analysis strategy tailored for smart grid control structure development. Control structures in electric power systems often span across several systems and stakeholders. Requirements elicitation for such control systems therefore requires coordination across many stakeholders and it is challenging to achieve a consistent design. To enable an iterative and distributed development we suggest a conflict management approach as a modular element of the design strategy, focusing on conflict identification and tracing. The idea is to describe a process starting from a tailored IEC 62559 template amended for recording controller conflicts and adapting the underlying use case management repository for collaborative work. Conflict identification is supported by Multilevel Flow Modeling providing abstracted conflict patterns.
\end{abstract}

Index Terms-ELECTRA IRP, Smart grids, Control, Conflict, Requirements, Modeling

\section{INTRODUCTION}

Control structures for the operation of electric power systems are often realized as ancillary services from generation or increasingly from demand response. Coordinated by system operators, they often span across several systems and stakeholders. The research project ELECTRA develops new control paradigms for operating European power systems with a scope beyond 2035. Ignoring the business-as-usual concepts of frequency and voltage control, this scope allows a greenfield approach in which control concepts can be conceived, developed and evaluated in lab trials [1]. As organizing principle, the future system is operated in a distributed architecture consisting in which grid regions are operated as Cells independently and coordinated by cell operators. The high level architectural concept, called Web-of-Cells, is summarized in [2]. A greenfield development with such a wide scope, tight coupling and the ambition of lab-based evaluation of the research needs to be supported with an appropriate requirements elicitation and management strategy. Such a requirements elicitation process via use cases, employing the IEC (International Electrotechnical Commission) 62559 template, and architecture modeling has been developed in earlier projects [3].

The ELECTRA project aims at developing novel monitoring, control and operator decision support systems. The definition of control functions and control structures imposes a special set of requirements for the generic use case method- ology. Whereas detailed specification of control functions and mechanisms are beyond the scope of the use case method, the general structure and distribution of control functions can be documented. A central aspect of this work is therefore to document the adaptation and usage of the IEC PAS (Public Available Specification) 62559 template.

Conflicting requirements for control structures can materialize in operational, but conflicting controllers. In more complex and distributed control situations, such as when several independent parties are involved this discovery may not be trivial, also as cross-domain phenomena may not be anticipated by domain experts. An approach for the identification of conflict patterns has been outlined in [4]. The authors of [4] suggest using the "Multi-level Flow Modeling" (MFM) language [5] as an intermediary functional representation. Given use cases with formal control domain annotation, a manual or semiautomated translation to MFM models is possible [6].

Another purpose of the adopted use case methodology is to formulate requirements for tracing them throughout the system development process and including testing. A formal way of tracing these requirements through development and lab testing supported using SysML [7] (Systems Modeling Language) as defined in the later sections of this contribution. Also here, reference to the control domain is critical in specifying test conditions. We chose to also adopt the SGAM (Smart Grid Architecture Model) from the EC (European Commission) M/490 mandate for our approach. The scope of this work is thus to adapt the existing methodology with the following features:

- Support for modeling control structures across systems,

- Enable conflict identification and tracking,

- Support requirements tracing for all layers and test stages. We aim to illustrate how these process requirements can be integrated with standard methods and tools by minimal adaptation of existing templates and a modular approach to altering the requirements evaluation process.

The paper is organized as follows. Section II introduces existing methods and basic concepts for the method to handle the documentation of conflicting controller requirements; the concepts of use case management, SGAM modeling, MFM and SysML-based requirements engineering are introduced. In Section III, the proposed process is described and put in context with the existing building blocks from the previous 
section. Section IV concludes the paper, outlining future work and next steps.

\section{Method BACKGROUND}

This section summarizes two established smart grid requirements engineering methods, Use Case management and SGAM, and introduces the two requirements engineering methods proposed to be employed in combination: MFM and SysML.

\section{A. Use Case management}

A central part of requirements management for Smart Grids focuses on so called use cases, which can serve as source and link for requirements [8]. Use Cases can capture the functionality of a system (and a system of systems) and respective interacting actors in the context of Smart Grids (which can for instance be other systems, applications, devices or people).

Within our ELECTRA approach, use cases are described in a semi-formal manner on the basis of an extended use case template from IEC 62559 adapted for ELECTRA and its scope. In the enterprise context, use cases can be developed to identify and define the relevant functionality within the scope of a particular enterprise, and thus limiting the various functions and related actors. The development can take place in an iterative process with two steps. In a first step, domain experts describe the main functionality and for instance involved actors. In a second step, ICT-experts (Information and Communication Technology) elaborate technical details, as recommended in [9], [10] and [11]. Finally, system components are identified and technical requirements for realization are being determined. These requirements contribute to the development of an ICT-architecture as well as its implementation and validation.

Instead of a greenfield approach for the development of use cases, existing external resources should be used and adapted for specific needs. An effort which addresses this has been performed in the standardization context by CENCENELEC-ETSI on behalf of the EC Mandate M/490 [12]. In the course of the work, generic high-level use cases (HLUC) which describe the most prominent Smart Grid functionality has been developed. They serve as a basis for the identification of finer functions and further architectural elements including their relations. These elements (e.g. components, businessand ICT-functions, information objects) are to be located in the SGAM model (see Section II-B). Based on the use cases and architectural elements, related ICT-standards as well as gaps in standardization can be identified. To do so, use cases are collected from different stakeholders, generalized and then linked with standards and classified, e.g. according to the SGAM or NIST Conceptual Model.

For a utility-specific ICT-architecture implementation, these generic use cases can support the identification of relevant standards and ICT-elements [9]. Also they can serve as input for the development of utility-specific use cases by adapting generic use cases coming from standardization according to the functional reference model. Besides the specific adaptation of generic use cases, detailed use cases can also be fed back to standardization bodies and, thus, influence standards. Mature use cases linked with standards can additionally be used to specify interoperability tests between systems.

With a large number of use cases elicited collaboratively by various experts collected in a Use Case Management Repository (UCMR), the complexity and the effort in coordination of the use case development increases. Moreover, use cases can be linked with further information, as for instance other use cases or references, so that many, not further integrated information sources are available.

\section{B. Smart Grid Architecture Model (SGAM)}

The SGAM has been developed in the course of the EU Mandate M/490 to European standardization organizations in order to support the Smart Grid deployment. It was developed as a part of the reference architecture framework during the work of the mandate. There, it is used to identify and characterize the power system domains, with their different zones, and the ICT systems which are relevant to this analysis with a special focus on interoperability/standardization. ICT systems must be described including the relevant technological components (such as storage, processing power and bandwidth) and the appropriate ICT objectives (such as resilience, maintenance, privacy and cyber-security, and interoperability) [13].

The SGAM defines a coordinated set of architecture viewpoints, informal concepts, as well as a method to map use case information to architectural artifacts and thus provides a structured approach for Smart Grid architecture development [14]. Key to the SGAM are its five interrelated interoperability viewpoints, addressing business, function, information, communication, and component concerns. These concerns can be traced back to the "Interoperability Framework" defined in [15] and are defined as interoperability layers in the SGAM. Each of these five layers further addresses organizational concerns and zones of power system management and relates them accordingly. Organizational concerns in this context are named "Domains" and defined according to the energy conversion chain/market roles: Generation, Transmission, Distribution, Distributed Energy Resources (DER), and Customer Premises. The zones of power system management include six hierarchical zones with decreasing distance to the physical network: Process, Field, Station, Operation, Enterprise, and Market.

Depicting the overall relationships of the interoperability layers, domains, and zones in the SGAM then provides a powerful tool to communicate about particular architectures on a high level. For that, the SGAM defines a five-layered, cube-like visualization. However, the whole SGAM framework neither provides formal models to start with nor information on the concrete application in a project context.

Tool support for the SGAM is crucial due to the complexity of its interrelated elements and development has already begun 
as can be seen in this paper. The extensions needed for toolchain integration enlarge the envisioned scope for the SGAM, making the methodology in conjunction with the IEC 62559 use case template easier to be put into daily utility practice, e.g. for IT/OT (IT and Operational Technology) integration [3]. To sustainably apply the SGAM in development projects, it needs to be incorporated into a tool-chain relying on standardized exchange formats. The SGAM can outgrow its original purpose, proving to be a methodology which can be utilized in different scenarios like IT/OT integration, innovative business models, security analysis, and architecture management.

\section{Functional Conflict Identification via MFM}

Key to the early control requirements analysis is a notation that represents the intended control function coupled with the relevant physical functions. Control intentions can either be derived from high-level operating goals, or as intermediate process objectives, to facilitate or enable operation of another process (e.g. keeping voltage at a generator bus constant to enable energy transfer). Multilevel Flow Modeling (MFM) [16] enables functional process representation at different abstraction levels and provides essential control functions and relations. MFM is formulated as diagrammatic notation combining the modeling concepts with syntactic and semantic rules for their interconnection. A particular MFM model expresses assumptions about behavioral roles, the relative topology and causality with respect to system objectives in scope. Such a model enables several forms of qualitative reasoning [17] about control situations with many applications to decision support and systems analysis [18]. A general introduction to MFM is found in [19], [20] and extensions for application to power systems and control structure analysis are presented in [21]-[23]. Tool support for MFM model includes graphical modeling and task dependent reasoning packages for modeling, diagnosis and operator support [18]. Model translation has been shown to be applicable [6].

In [4], it has been shown that MFM models are suitable to identify several types of functional controller conflict on early requirements elicitation. Following [4], controller conflict is the phenomenon in which the activation of one control structure has undesired effects on one or several other control structures. In addition to the pure goal conflicts addressed in other work on conflicting goals, such as [24], MFM is suitable to identify also conflict patterns within the process using the semi-formal approach outlined in [4].

\section{Requirements tracing with SysML}

Systems engineering is generally considered a multidisciplinary and holistic approach [25] to cope with the development of solutions for complex engineering problems. To deal with the complexity of such a holistic approach, the concepts for model-based systems engineering have been developed by the OMG (Object Management Group). One of the underlying pre-conditions is that a general-purpose methodology and modeling language to cope with such a problem class must include the phases of specification, design, analysis and verification of the system as well as hardware, equipment, software, data and procedures. For obvious reasons, this is also a problem class adressed by the ELECTRA project consortium to be tackled with. original domains intended by the OMG were aerospace, automative and health care. The OMG SysML is an extension of the Unified Modeling Language (UML) and its version 2, which can be considered the de-facto standard for software modeling and is used in context with the IEC 62559 template. One particular aspect in the scope of this contribution is the introduction of new Meta-Object based diagram types (MOF) to standard UML modeling [7].

Within the IEC 62559 IntelliGrid method, the structural and behavioral aspects of a technical Smart Grid solution are already covered. In addition, the annexes of the 62559 provide a thorough overview on how to document non-functional requirements and parameters for a technical smart grid solution. The UML lacks appropriate formal diagrams for those two important aspects from IEC 62559. Up till the introduction of this diagram type, only functional dependencies could be modeled in UML. Apart from the actual requirements, stereotypes for modeling test cases, activities and rationales are provided. We suggest to use those new types of diagrams in accordance with the IEC 62559 as it provides from the Model Based System Engineering (MBSE) view a way to achieve traceability.

\section{Proposed Process}

The methods reviewed in the previous section are integrated in a partly iterative process which picks up existing ELECTRA IRP inputs. An overview of this process is sketched in Fig. 1.

\section{A. Project-specific context}

The proposed process takes various inputs from the ELECTRA IRP, as summarized in the following paragraphs. The aim is to gather all this information in structured self-contained use case documents in an online repository. The following items are therefore inputs to template, UCMR and SGAM designation system.

1) Inputs to Actor Model: Fundamental ELECTRA concepts for describing systems and business roles are the terms agreed upon in the glossary of ELECTRA, defining actor types and systems, which will be re-used in the UCMR.

Next, the web-of-cells control architecture requires that control functions are distributed over several control systems, and these systems need to be identified to describe the allocation of a distributed control structure to separate actors. Designating the conceptual location of these systems is achieved by defining control topology levels, organizing the domain into levels along the value chain of control systems: CTL-3 Cell Group; CTL-2 Cell; CTL-1 Aggregated Flexibility; CTL-0 Device.

Critical to the description of control functions is a specification of actor associations with the control domain, which is characterized by

a) The Single Reference Power System (SRPS), a conceptual model of a web-of-cells power system aligned with a set of concrete example grid models, which are necessary for 


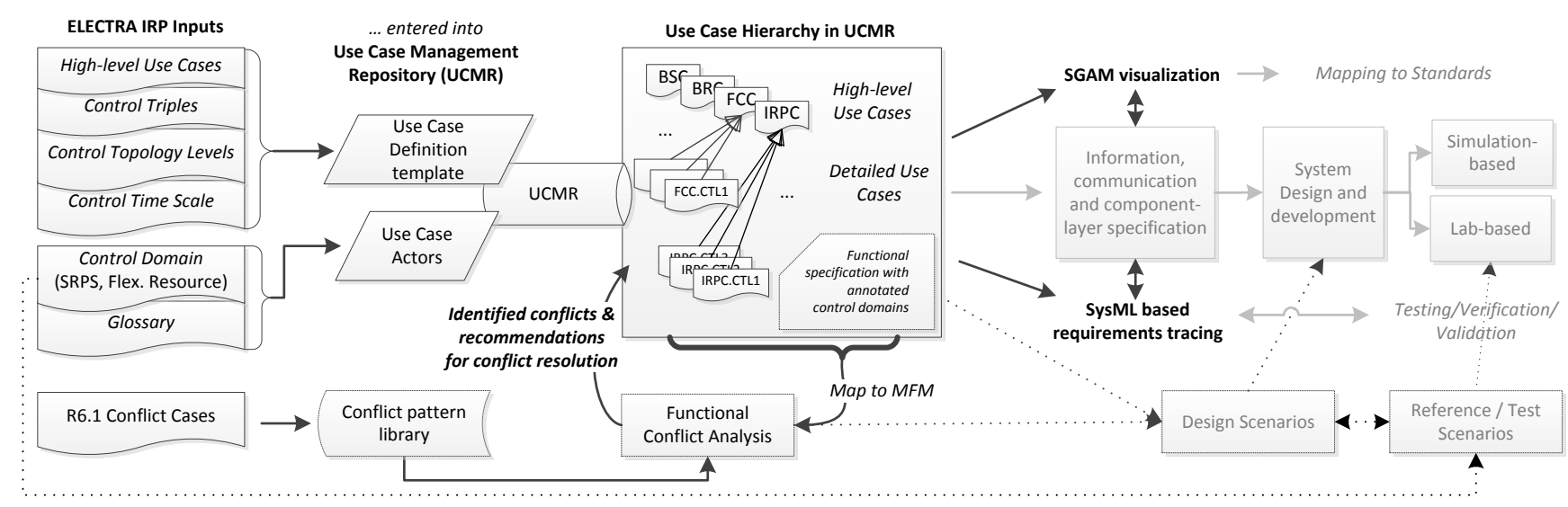

Fig. 1. Proposed overall ELECTRA requirements process: Project specific inputs are listed on the left; the central process elements (printed in bold) are recorded in the UCMR; systematic control domain and actor modeling enable functional conflict identification; finally SGAM reference designation and SysML annotations then enable visual inspection and requirements tracing toward lab testing. Dotted lines indicate information re-use by control domain annotation.

referencing the definition of control objectives, system inputs and observables; and

b) Flexibility Resources which serve as actuators providing the system inputs (systems services).

2) Inputs to Use Case Definition: As starting point, a high-level description outlining the key architectural concepts have been defined by the corresponding stakeholders and are available as High-level use cases (HLUCs). Apart from highlevel use cases, other technical information is available to the modelers regarding context and mechanism specifications for characterizing the control functions and domain:

- The ELECTRA concept of control triples defines the technical controller scope as a set of: control aim/objective, system input variable and observable;

- Control time scales (CTS-1 .. CTS-3) define characteristic groups of requirements for control delays and response times;

- Control mechanisms, or rather principles of controller coordination, defining the principle employed for the coordination of control functions across use cases;

- Optimality criteria are associated with each control objective.

These concept introductions are brief due to page limitations here; for a thorough introduction we refer to the forthcoming extensive report which will be provided in the form of a deliverable of the ELECTRA IRP.

\section{B. Template adaptation and UCMR input format}

The control functions to be defined by detailed use cases are identified as a) specialization of a single HLUC, and by selecting b) a single control topology level (CTL-x) for each use case. This rule generates a nominal hierarchy of controller use cases. Technical input for use cases writing is compiled by grouping the technical features listed above. Use case writing then consists in one part of mapping this information into the use case template. Here we outline this proposed mapping and motivate required (minimal) adaptations of the template.
The requirements structure of use cases is geared toward ICT system specification, which includes views not commonly employed for control systems specification. Therefore, the compiled technical descriptions need to be supplemented with ICT specific views. For the time being, no detailed evaluation can be provided for this work in progress, therefore this paper focusses on the proposed methodology; an example by the use case team from the ELECTRA IRP is made available in [26].

1) Actors Definition and Grouping: Hierarchical consistency of control structures and systems within the Web-ofCells concept is achieved by a structured modeling of use case actors via role models and explicit control domain relations. The respective concepts are noted in Section III-A1. These entities can be mapped on domains and zones for an adopted SGAM model as well as to use role concepts and provide ways to properly group the actors that have been defined in ELECTRA.

In the UCMR, these well-specified actors can then be associated with use cases via their role in the "step by step analysis". A use case specific grouping of actors can be formulated in template Section 3 (cf. Section V).

2) Adaptation of IEC 62559 Use Case template: One of the first steps to properly cope with the new requirements imposed by the ELECTRA IRP is to adapt the use of individual fields in the IEC 62559 template.

An important issue raised here, was the aspect of semantically changing the standard, e.g. changing the use of individual labels and fields. From the ELECTRA point of view, we could consider leaving fields out, adapting their use and adding new ones as valid approaches to deal with the application of the IEC 62559-2 template. Within the IEC 62559-2 template, no label shall be deemed mandatory, but it may be argued that many labels from template Sections 1 and 2 shall be included to preserve the use case key components and due to best practice. As for our work, we have chosen a) to focus on interpreting existing fields in a specialized form suited for the ELECTRA context, and b) to adapt fields or add new ones in rare cases where the required scope is extended beyond the 
purpose of the original template.

The following summarizes the mapping of ELECTRA and control specific information to the template (see Section V for an outline of the template, and [26] for the full template).

- Systematic identifiers for the use cases defined in Section 1.1 "Name of the Use Case";

- Concept of Control Triples: to be dealt with in the template at objectives (1.3) and use case conditions (1.6);

- The control time scale is annotated as "Requirement" (Section 6) and identified via drag\&drop to Section 4 "Step by Step analysis" in the UCMR;

- Optimality criteria are equivalent to KPI (Section 1.5);

- ELECTRA Vertical/Horizontal control coordination are recorded under Section 1.7 "Further Information" based on a taxonomy of control mechanisms.

The following new fields have been identified as needed for the work in ELECTRA:

- Amendment of Section "1.3 Scope and Objectives" with field "Control Domain";

- To record the results of conflict analysis, see following subsection, a new Section 9 "Controller Conflicts and Misuse Cases" was added to the template.

\section{Conflict Identification and Feedback}

The early identification of conflicting requirements is an important contribution in requirements engineering [27]. Whereas many methods for identification of requirements conflicts have been proposed in the literature, few are specialized for control conflicts. The proposed process of conflict identification is outlined in Fig. 2 of this contribution. The individual steps will be presented in the following.

1) Controller Conflict and Conflict Patterns: In case of control requirements the focus is typically on identifying and resolving goal conflicts [24]. However, also for control systems, conflicts may appear with respect to both goals and resources at all implementation layers. Motivated by SGAM interoperability layers, may be defined conflict classes: business (goals,responsibility), functional (objectives, degrees of freedom), information (interpretation), communication (e.g. interacting channels), and process (physical/undesired interactions). In particular business, functional and process level conflicts are well suited to be identified or anticipated at an early requirements stage [4]. A recent ELECTRA IRP internal report (R6.1 sec.5.1) identified 24 cases of controller conflict reported in the literature, most of which are functional conflicts. Following the method outlined in [4], a library of MFM conflict patterns will be established based on observed conflict in the literature.

2) Conflict Identification: For each control domain an MFM model shall be generated, integrating the control functions of all use cases referring to the same or an overlapping domain. Use case objectives, actors, time scales and control domain specification will be sourced establish an accurate model. By comparison of this model with the patterns library matching conflict patterns will be identified.
3) Feedback and Conflict Table: Conflict patterns are stereotypical cases for which prior information on phenomena for testing as well as potential conflict resolution strategies are known. Such information can be provided as feedback into the UCMR. The use case template and UCMR is therefore amended with a Section 9, containing a table to record identified Controller Conflicts, using the following fields: Id; Name; Description; Conflict References; Related-Actors / Use Cases / Requirements / Objectives; Recommended Mitigation; Status.

At present the table fields are kept as free text, and are also free to be edited during the use case writing process, e.g. to record anticipated conflicts for later follow-up.

\section{SGAM Visualization}

One way to discuss about the system solution is based on visuals instead of the pure text template for the use case. Most of the time, people think visual and the actual content can be discussed better. In ELECTRA, we re-use an existing 3D visualization for the SGAM methods.
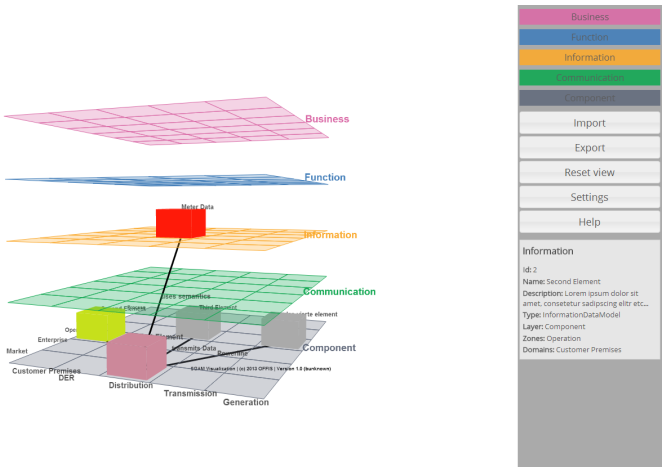

Fig. 2. SGAM 3D Visualiser

As the SGAM domains axis defines a different view of the power system organizational concerns than proposed in ELECTRA Cell architecture. For the SGAM, a new domain axis which better fits the use of SGAM in ELECTRA will be defined. The focus will change from generation and transmission to Cell oriented concepts. Adaptation of zone/domain annotations in the UCMR and use case template are straightforward. Based on the proposed changes to the domain and zone of SGAM, the visuals can be adopted to reflect the new focus of the controller conflict handling.

\section{E. Requirements Tracing}

The introduction of SysML to the existing tool chain provides a way to properly model non-functional requirements in a visual way. The XMI serialization can be used in accordance with formats like RIF (Requirements Interchange Format) to re-use the requirements in development tools. In addition, the visuals mainly motivate the traceability using the requirements diagram, so both a formal and informal way to trace objectives is achieved. 


\section{CONCLusion}

This contribution presented a way to adopt the existing SGAM and IntelliGrid methodology and processes for Smart Grid Architecture Modeling to the scope of systems engineering for Smart Grids at the solution level. For ELECTRA, an approach to adopt the tools Use case, UCMR, SGAM and visuals was proposed and introduced. Based on this initial methodology, extensions and changes have been proposed to adapt to the ELECTRA scope and to identfify and trace controller conflict. In view of control structure requirements, the need for systematic control domain modeling is common across methods and key to enabling a structured conflict identification. Further work will entail the evaluation of the proposed approach within the ELECTRA project.

\section{Appendix: Outline of IEC 62559-2 template}

For reference, an outline of the use case template is provided. The full template with comments and example text is available online [26].

1 Description of the use case

Subsections: 1.1 Name of use case; 1.2 Version management; 1.3 Scope and objectives of use case; 1.4 Narrative of use case; 1.5 Key performance indicators (KPI); 1.6 Use case conditions; 1.7 Further information; 1.8 General remarks

2 Diagrams of use case

3 Technical details

Subsections: 3.1 Actors; 3.2 References

4 Step by step analysis of use case

Subsections: 4.1 Overview of scenarios; 4.2 Steps Scenarios

5 Information exchanged

6 Requirements

7 Common Terms and Definitions

8 Custom information (optional)

*9 Controller Conflicts and Misuse Cases

Section 9 is an amendment proposed in this work.

\section{ACKNOWLEDGMENTS}

The research leading to these results has received funding from the European Union Seventh Framework Programme [FP7/2007-2013] under grant agreement $\mathrm{n}^{\circ} 609687$, and includes support from the ELECTRA REX researcher exchange programme.

\section{REFERENCES}

[1] L. Martini, L. Radaelli, H. Brunner, C. Caerts, A. Morch, S. Hänninen, and C. Tornelli, "Voltage and frequency control for future power systems: the electra irp proposal," in Proceedings of the 23rd International Conference on Electricity Distribution. Lyon: CIRED, June 2015.

[2] R. Dh́ulst, J. Merino Fernandez, E. Rikos, D. Kolodziej, K. Heussen, D. Geibel, A. Temiz, and C. Caerts, "Voltage and frequency control for future power systems: the electra irp proposal," in Proceedings of the 2015 International Symposium on Smart Electric Distribution Systems and Technologies (EDST). Vienna: IEEE, 2015.

[3] J. Trefke, S. Rohjans, M. Uslar, S. Lehnhoff, L. Nordström, and A. Saleem, "Smart Grid Architecture Model Use Case Management in a large European Smart Grid Project," in 4th IEEE European Innovative Smart Grid Technologies (ISGT), 2013.

[4] K. Heussen, O. Gehrke, and H. Niemann, On Early Conflict Identification by Requirements Modeling of Energy System Control Structures. IEEE, 2015.
[5] M. Lind, "Multilevel flow modelling of process plant for diagnosis and control," in Proc. International Meeting on Thermal Reactor Safety, 1982.

[6] K. Heussen, J. Weckesser, and D. Kullmann, Pattern-based Automatic Translation of Structured Power System Data to Functional Models for Decision Support Applications. IEEE, 2013.

[7] T. Weilkiens, Systems Engineering with SysML/UML. OMG Group, 2008.

[8] J. Trefke, J. M. González, and M. Uslar, "Smart Grid Standardisation Management with Use Cases," in 2nd IEEE ENERGYCON Conference \& Exhibition, Florence, Italy, 2012, pp. 966-971.

[9] C. Dänekas, C. Neureiter, S. Rohjans, M. Uslar, and D. Engel, "Towards a model-driven-architecture process for smart grid projects," in Digital Enterprise Design \& Management, ser. Advances in Intelligent Systems and Computing, P. Benghozi, D. Krob, A. Lonjon, and H. Panetto, Eds. Springer International Publishing, 2014, vol. 261, pp. 47-58.

[10] F. Andren, T. Strasser, S. Rohjans, and M. Uslar, "Analyzing the Need for a Common Modeling Language for Smart Grid Applications," in 11th IEEE International Conference on Industrial Informatics, 2013.

[11] M. Uslar, F. Andren, W. Mahnke, S. Rohjans, M. Stifter, and T. Strasser, "Hybrid grids: Ict-based integration of electric power and gas grids-a standards perspective," in Innovative Smart Grid Technologies (ISGT Europe), 2012 3rd IEEE PES International Conference and Exhibition on. IEEE, 2012, pp. 1-8.

[12] Smart Grid Coordination Group, "Smart Grid Reference Architecture," CEN-CENELEC-ETSI, Tech. Rep., 2012.

[13] X. Fang, S. Misra, G. Xue, and D. Yang, "Smart Grid - The New and Improved Power Grid: A Survey," IEEE Communications Surveys \& Tutorials, vol. 14, no. 4, pp. 944-980, 2011.

[14] M. Uslar, M. Specht, C. Dänekas, J. Trefke, S. Rohjans, J. M. González, C. Rosinger, and R. Bleiker, Standardization in Smart Grids: Introduction to IT-Related Methodologies, Architectures and Standards. Springer Science \& Business Media, 2012.

[15] The GridWise Architecture Council, "GridWise Interoperability ContextSetting Framework," Tech. Rep., 2008. [Online]. Available: http: //www.gridwiseac.org/pdfs/interopframework $\searrow$ v1 $\backslash \_1 . p d f$

[16] M. Lind, "Modeling goals and functions of complex industrial plant," Applied Artificial Intelligence, vol. 8, no. 2, pp. 259-283, 1994.

[17] $\longrightarrow$, "Reasoning about Causes and Consequences in Multilevel Flow Models," in ESREL 2011, Troyes, France, 2011.

[18] K. Heussen and M. Lind, "On support functions for the development of mfm models," in Proceedings of STSS 2012, Okayama, 2012.

[19] M. Lind, "An introduction to multilevel flow modelling," International Journal of Nuclear Safety and Simulation, vol. 2, no. 1, 2011.

[20] - "Control Functions in MFM: Basic principles," International Journal of Nuclear Safety and Simulation, vol. 2, no. 2, 2011.

[21] K. Heussen, A. Saleem, and M. Lind, "Control architecture of power systems: Modeling of purpose and function," in Proceedings of the IEEE PES General Meeting 2009, 2009.

[22] K. Heussen and M. Lind, "Representing causality and reasoning about controllability of multi-level flow-systems," in Systems Man and Cybernetics (SMC), 2010 IEEE International Conference on, oct. 2010, pp. $1896-1903$

[23] K. Heussen, "Control architecture modeling for future power systems," Ph.D. dissertation, Technical University of Denmark, 2011.

[24] I. A. El-Maddah and T. S. Maibaum, "The gopesd tool: An integrated development environment for process control requirements and design," in Fundamental Approaches to Software Engineering. Springer, 2004, pp. 261-266.

[25] S. Friedenthal, A. Moore, and R. Steiner, A practical Guide to SysML - The Systems Modeling language. OMG Group, 2014.

[26] M. U. Kai Heussen, Carlo Tornelli. (2015, 07) Edst 2015 electra use case template for conflict analysis. Researchgate.Net. ELECTRA IRP FP 7. 10.13140/RG.2.1.2951.4088. [Online]. Available: 10.13140/RG.2.1.2951.4088

[27] A. Van Lamsweerde, R. Darimont, and E. Letier, "Managing conflicts in goal-driven requirements engineering," Software Engineering, IEEE Transactions on, vol. 24, no. 11, pp. 908-926, 1998. 